\title{
Photoacoustic image reconstruction with uncertainty quantification
}

\author{
J. Tick ${ }^{1}$, A. Pulkkinen ${ }^{1}$ and T. Tarvainen ${ }^{1,2}$ \\ ${ }^{1}$ Department of Applied Physics, University of Eastern Finland, P.O.Box 1627, 70211 Kuopio, Finland \\ 2 Department of Computer Science, University College London, Gower Street, London WC1E 6BT, United Kingdom
}

\begin{abstract}
Photoacoustic tomography is a hybrid imaging technique that has various applications in biomedicine. In a photoacoustic image reconstruction problem (inverse problem), an initial pressure distribution is reconstructed from measured ultrasound waves which are generated by the photoacoustic effect induced by an optical excitation. In this work, the image reconstruction problem is approached in the framework of Bayesian inversion. The approach is tested with three dimensional numerical simulations. The initial pressure distribution is reconstructed in full-view and limited-view setups. In addition, the reliability of the obtained estimates is assessed. The numerical studies show that accurate estimates of the initial pressure distribution and uncertainty information can be obtained utilizing Bayesian approach.
\end{abstract}

Keywords - photoacoustic tomography, inverse problems, Bayesian methods, uncertainty quantification

\section{INTRODUCTION}

Photoacoustic tomography (PAT), also known as optoacoustic tomography, is a hybrid imaging modality that is characterized by high contrast and resolution $[1,2,3]$. PAT can provide structural, functional, and molecular information. Due to its many attractive features, its applications include e.g. detection of skin and breast cancer, imaging of vascular system and small animal imaging [1].

In PAT, a short pulse of visible or near-infrared light is used to irradiate the imaged object. Due to the photoacoustic effect, ultrasound waves are generated. The waves propagate to the surface of the object, where they are measured. From these time-varying measured pressure waves, an initial pressure distribution is reconstructed. This is also known as the acoustic inverse problem of PAT and it has been widely studied, see e.g. $[4,5,6]$ and the references therein.

The initial pressure distribution can be estimated using variety of reconstruction algorithms. Commonly used reconstruction algorithms are backprojection [7, 8, 9, 10], time reversal $[11,12,13]$ and model based inversion approaches $[14,15,16,17,18,19]$. The backprojection algorithms are based on analytical inversion formulae and are analogues to the Radon transform. The time reversal algorithms perform image reconstruction by simulating ultrasound propagation backwards in time. In the model-based methods, the initial pressure distribution is obtained by minimizing the error between the measured signals and the signals computed by the photoacoustic forward model.

Recently, PAT image reconstruction method utilizing a Bayesian approach was proposed [20]. The approach provides the estimates of the initial pressure distribution together with information about the reliability of these estimates. In this work, the approach is extended to three dimensions (3D). A matrix free method utilizing the adjoint of the forward operator is implemented. The approach is investigated using numerical simulations in different sensor geometries.

\section{Photohcoustic Model}

Absorption of the energy of a short light pulse into the object causes thermoelastic expansion of the object. This in turn leads to a generation of a pressure wave which propagates through the object. Propagation of the pressure waves in a linear and homogeneous medium can be described by wave equation

$$
\begin{aligned}
\left(\frac{\partial^{2}}{\partial t^{2}}-c^{2} \nabla^{2}\right) p(r, t) & =0 \\
p(r, t=0) & =p_{0}(r) \\
\frac{\partial}{\partial t} p(r, t=0) & =0
\end{aligned}
$$

where $p$ is the pressure, $r$ is the spatial position, $t$ is the time, $c$ is the speed of sound and $p_{0}$ is the initial pressure distribution [4]. The pressure waves $p(r, t)$ can be measured on the surface of the object as a function of time.

In practice, the measured pressure waves are polluted with noise. A common approach is to assume the noise being additive. The discrete observation model with an additive noise model for PAT is

$$
p_{t}=K p_{0}+e,
$$

where $p_{t}$ is a vector composed of measured acoustic pressure waves, $p_{0}$ is the discrete initial pressure distribution, $K$ is the discretized forward model (1) which maps the initial pressure distribution to the measurable data, and $e$ denotes the noise. 


\section{BAYESIAN APPROACH}

In the inverse problem of PAT, the initial pressure distribution $p_{0}$ is reconstructed from the detected pressure signals $p_{t}$. In this work, the image reconstruction is performed utilizing the Bayesian approach [20, 21]. In the Bayesian approach, all parameters are treated as random variables. The solution of the inverse problem, the posterior density, is obtained based on the model, prior information and measurements.

Let the noise be normally distributed $e \sim \mathscr{N}\left(\eta_{e}, \Gamma_{e}\right)$ where $\eta_{e}$ and $\Gamma_{e}$ are the mean and the covariance of the noise. Similarly, the initial pressure distribution is assumed to be normally distributed $p_{0} \sim \mathscr{N}\left(\eta_{p_{0}}, \Gamma_{p_{0}}\right)$ where $\eta_{p_{0}}$ and $\Gamma_{p_{0}}$ are the mean and covariance of the prior, and independent of the noise. With these assumptions, the posterior distribution of the observation model (2) can be expressed as a Gaussian distribution $p_{0} \mid p_{t} \sim \mathscr{N}\left(\eta_{p_{0} \mid p_{t}}, \Gamma_{p_{0} \mid p_{t}}\right)$ with mean [20]

$$
\eta_{p_{0} \mid p_{t}}=A^{-1} b
$$

and covariance

$$
\Gamma_{p_{0} \mid p_{t}}=A^{-1}
$$

where

$$
\begin{aligned}
A & =K^{\mathrm{T}} \Gamma_{e}^{-1} K+\Gamma_{p_{0}}^{-1}, \\
b & =K^{\mathrm{T}} \Gamma_{e}^{-1}\left(p_{t}-\eta_{e}\right)+\Gamma_{p_{0}}^{-1} \eta_{p_{0}} .
\end{aligned}
$$

Reconstructed image is obtained by calculating point estimates of the posterior density. In this work, maximum a posteri (MAP) estimate is considered. In a Gaussian case, the MAP estimate is the mean of the posterior distribution $p_{0, \mathrm{MAP}}=\eta_{p_{0} \mid p_{t}}$. The reliability of the reconstructed images can be assessed by computing the credibilities of the estimates. In this work, the marginal densities of the posterior distribution are determined. These can be obtained from the diagonal of the posterior covariance matrix $\Gamma_{p_{0} \mid p_{t}}$.

Computation of the MAP estimate and posterior covariance in the matrix form can become impractical in three dimension, since storage and inversion of very large matrices are required. Therefore, a matrix free method is used in this work. The method utilizes the adjoint of the forward operator implemented with the k-Wave toolbox [22]. Furthermore, the MAP estimate and the posterior covariance are determined iteratively using iterative solvers of MATLAB.

\section{NUMERICAL SIMULATIONS}

The approach was investigated with numerical simulations. The simulation domain was a cube with a side length of $10 \mathrm{~mm}$ and it was discretized by $306^{3}$ cubic voxels with the side length of $\Delta h=32.7 \mu \mathrm{m}$. The medium was assumed to be non-attenuating with a constant speed of sound $c=1500 \mathrm{~m} / \mathrm{s}$. The simulated initial pressure distribution of the medium consisted of nine spheres with the radius of $1.43 \mathrm{~mm}$ and initial pressure $p_{0}=5$. Eight of these spheres were located in the corners of the cube and one sphere was located in the middle of the cube. The ambient initial pressure distribution had a value of zero.

The data was simulated using the $k$-space time domain method implemented with the k-Wave MATLAB toolbox [23]. The pressure signals were recorded for $14.1 \mu \mathrm{s}$ at sampling frequency of $60 \mathrm{MHz}$ (849 temporal samples) in a fullview sensor geometry and two limited-view sensor geometries. In many practical applications of PAT such as imaging of skin, the pressure signals cannot be recorded around the whole object (full-view sensor geometry) which results in a limited-view data collection. In the full-view setup (6-side), 62119 acoustic sensors were located at all sides of the cube. In the first limited-view sensor geometry (L-shape), 20808 sensors were located on two adjacent sides (sides in the direction of the negative $y$ and positive $z$ axes) of the cube. In the second limited-view setup (1-side), 10404 sensors were located only on one side (side in the direction of the positive $z$ axis) of the domain. The uncorrelated Gaussian distributed noise with a zero mean and a standard deviation proportional to $1 \%$ of the peak amplitude of the simulated pressure signal noise was added to the simulated pressure signals.

In the image reconstruction, the discretization of the domain consisted of $204^{3}$ cubic voxels with the width of $\Delta h=$ $49.0 \mu \mathrm{m}$. As prior information, a white noise prior was used. White noise prior is described by the mean $\eta_{p_{0}}$ and the covariance matrix $\Gamma_{p_{0}}=\operatorname{diag}\left(\sigma_{\mathrm{p}_{0}}^{2}\right)$. In this work, the mean $\eta_{p_{0}}=0$ and standard deviation $\sigma_{p_{0}}=2$ was utilized. The measurement noise was considered to be uncorrelated Gaussian distributed noise with zero mean and the standard deviation set to $1 \%$ of the peak positive amplitude of the noisy simulated data. The initial pressure distributions were estimated by computing the MAP estimate (3), and marginal densities were determined in selected points inside the domain to assess the reliability of the obtained estimates. All the computations were performed on a GPU.

\section{A. Results}

Fig. 1 shows the simulated and estimated initial pressure distributions. As it can be seen, the estimated initial pressure distribution looks qualitatively very similar to the original distribution in the 6-side setup. Visually the quality of the estimated initial pressure distribution is reduced when the limited-view sensor geometries are used. The quality reduc- 


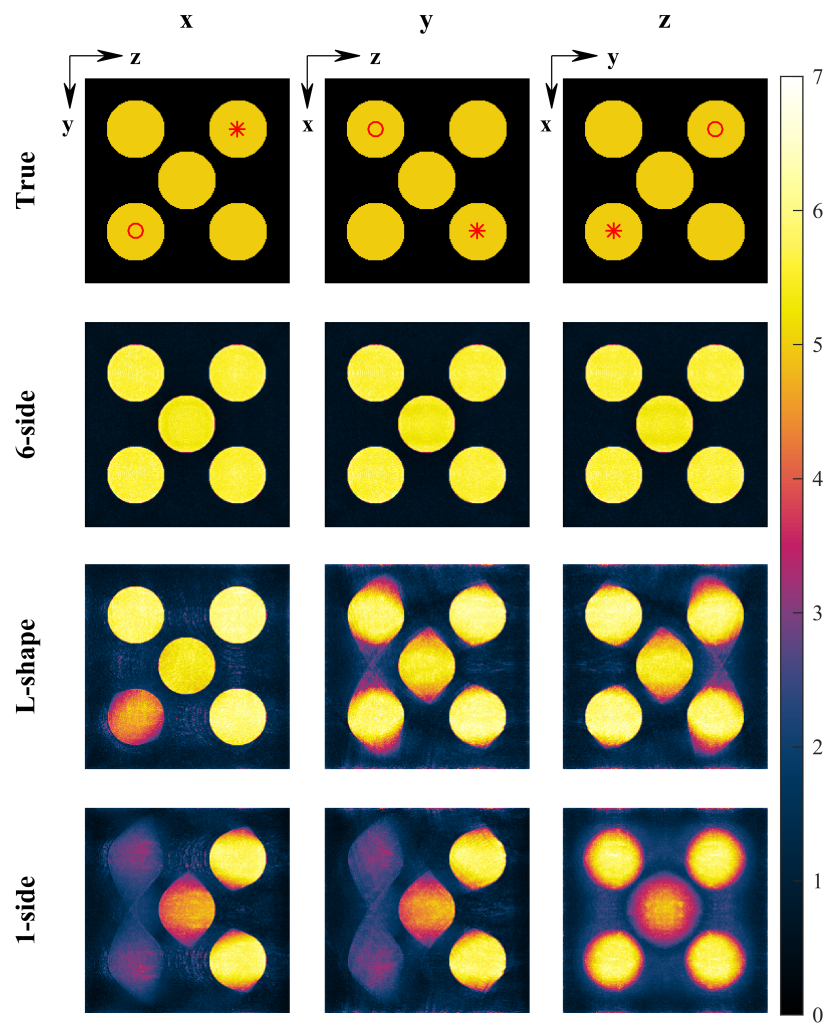

Fig. 1: Maximum intensity projections of the initial pressure distribution in $x, y$ and $z$ directions for the simulated distribution (first row) reconstructions in the 6-side setup (second row), reconstructions in the L-shape setup (second row) and reconstruction in the 1-side setup (fourth row). The asterisks and circles indicate the two locations where the marginal densities are plotted.

tion is more severe far from the sensor surface, and artefacts and distortions can be seen in those areas. In the L-shape sensor geometry, this can be observed for example in the leftlower corner of the maximum intensity projection in $x$ direction and in the left side of the maximum intensity projection in $y$ direction. Similarly, in the 1-side sensor geometry, quality reduction can be seen for example in the left side of the maximum intensity projections in $x$ and $y$ directions. Comparing the estimates obtained using different limited-view sensor geometries, it can be seen that the estimated initial pressure distributions in L-shape sensor geometry looks qualitatively better than the estimate obtained using the 1-side setup.

Fig. 2 shows the marginal densities at two points inside the domain indicated in Fig. 1. As it can be seen, the maximum of the marginal density is not necessarily at the location of the true value, but the true value seems to be within the principal support of the distribution in all cases apart from the marginal density computed in the point indicated with as-
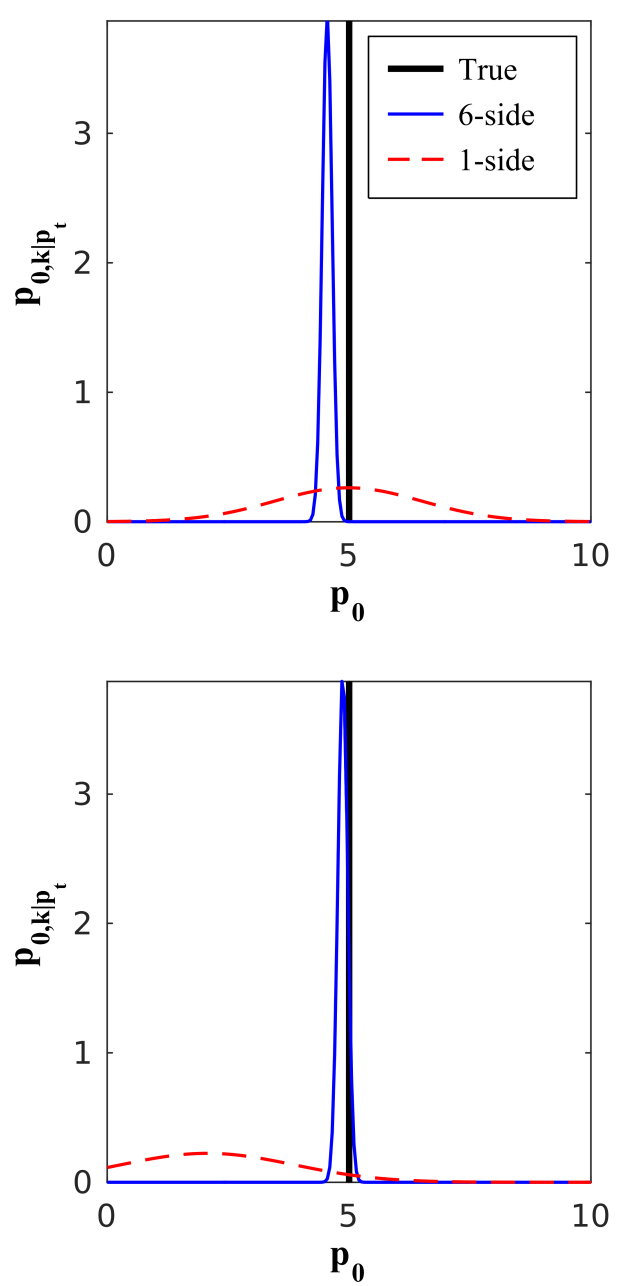

Fig. 2: Marginal densities of the posterior distribution obtained using the 6-side (blue solid line) and the 1-side sensor geometry (dashed red line). In the top figure, the marginal density is computed in the location which is indicated in Fig. 1 with an asterisk. In the bottom figure, the marginal density is computed in the location which is indicated in Fig. 1 with a circle. In both points, the true initial pressure $p_{0}=5$ (vertical black line).

terisk using the 6-sided sensor geometry. Furthermore, the marginal density is narrower in the 6-side sensor geometry than in the 1-side sensor geometry. This demonstrates that the uncertainty of the estimate obtained using 6-side sensor geometry is smaller than the uncertainty of the estimate obtained using 1-side sensor geometry, as should be expected due to the lower quality sensor setting 


\section{CONCLUSIONS}

The Bayesian approach to photoacoustic tomography was considered and extended to 3D. A matrix free method was implemented, and image reconstruction and estimating the reliability of the images was investigated. The numerical simulations showed that the sensor geometry affected both the estimates and their uncertainties. The quality of the images reduced and the uncertainty increased as the sensor geometry turned into limited-view. Overall, the results show that the Bayesian approach can be used to provide accurate estimates of the initial pressure distribution as well as information about the uncertainty of the estimates. However, more research is required for the interpretation of when uncertainty estimates can be regarded safe, see e.g. [24].

\section{ACKNOWLEDGEMENTS}

The authors would like to thank Felix Lucka (University College London) for the help with the adjoint implementation. The Tesla K40 GPU used for this research was donated by the NVIDIA Corporation. This work has been supported by the Academy of Finland (projects 286247 and 250215 Finnish Centre of Excellence in Inverse Problems Research), Instrumentarium Science Foundation, Magnus Ehrnrooth Foundation, and Jane and Aatos Erkko Foundation.

\section{CONFLICT OF INTEREST}

The authors declare that they have no conflict of interest.

\section{REFERENCES}

1. Beard P.. Biomedical photoacoustic imaging Interface Focus. 2011;1:602-631.

2. Li C., Wang L. V.. Photoacoustic tomography and sensing in biomedicine Physics in Medicine and Biology. 2009;54:R59-R97.

3. Lutzweiler C., Razansky D.. Optoacoustic imaging and tomography: Reconstruction approaches and outstanding challenges in image performance and quantification Sensors (Switzerland). 2013;13:7345-7384.

4. Xu M., Wang L. V.. Photoacoustic imaging in biomedicine Review of Scientific Instruments. 2006;77:041101.

5. Kuchment Peter, Kunyansky Leonid. Mathematics of thermoacoustic tomography European Journal of Applied Mathematics. 2008;19:191224.

6. Rosenthal A., Ntziachristos V., Razansky D.. Acoustic inversion in optoacoustic tomography: A review Current Medical Imaging Reviews. 2013;9:318-336.

7. Xu M., Xu Y., Wang L. V.. Time-domain reconstruction algorithms and numerical simulations for thermoacoustic tomography in various geometries IEEE Transactions on Biomedical Engineering. 2003;50:1086-1099.

8. Xu M., Wang L. V.. Universal back-projection algorithm for photoacoustic computed tomography Physical Review E - Statistical, Nonlinear, and Soft Matter Physics. 2005;71:016706.
9. Kunyansky L. A.. Explicit inversion formulae for the spherical mean Radon transform Inverse Problems. 2007;23:373-383.

10. Finch D., Patch S. K., Rakesh . Determining a function from its mean values over a family of spheres SIAM Journal on Mathematical Analysis. 2004;35:1213-1240.

11. Xu Y., Wang L. V.. Time Reversal and Its Application to Tomography with Diffracting Sources Physical Review Letters. 2004;92:339021339024.

12. Burgholzer P., Matt G. J., Haltmeier M., Paltauf G.. Exact and approximative imaging methods for photoacoustic tomography using an arbitrary detection surface Physical Review E - Statistical, Nonlinear, and Soft Matter Physics. 2007;75:046706.

13. Hristova Y., Kuchment P., Nguyen L.. Reconstruction and time reversal in thermoacoustic tomography in acoustically homogeneous and inhomogeneous media Inverse Problems. 2008;24:055006.

14. Deán-Ben X. L., Buehler A., Ntziachristos V., Razansky D.. Accurate model-based reconstruction algorithm for three-dimensional optoacoustic tomography IEEE Transactions on Medical Imaging. 2012;31:1922-1928.

15. Rosenthal A., Razansky D., Ntziachristos V.. Fast semi-analytical model-based acoustic inversion for quantitative optoacoustic tomography IEEE Transactions on Medical Imaging. 2010;29:1275-1285.

16. Deán-Ben X. L., Ntziachristos V., Razansky D.. Acceleration of optoacoustic model-based reconstruction using angular image discretization IEEE Transactions on Medical Imaging. 2012;31:1154-1162.

17. Zhang J., Anastasio M. A., Rivire P. J. La, Wang L. V.. Effects of different imaging models on least-squares image reconstruction accuracy in photoacoustic tomography IEEE Transactions on Medical Imaging. 2009;28:1781-1790

18. Paltauf G., Viator J. A., Prahl S. A., Jacques S. L.. Iterative reconstruction algorithm for optoacoustic imaging Journal of the Acoustical Society of America. 2002;112:1536-1544.

19. Wang K., Su R., Oraevsky A. A., Anastasio M. A.. Investigation of iterative image reconstruction in three-dimensional optoacoustic tomography Physics in Medicine and Biology. 2012;57:5399-5423.

20. Tick Jenni, Pulkkinen Aki, Tarvainen Tanja. Image reconstruction with uncertainty quantification in photoacoustic tomography The Journal of the Acoustical Society of America. 2016;139:1951-1961.

21. Kaipio Jari, Somersalo Erkki. Statistical and Computational Inverse Problems. Springer Science \& Business Media 2006.

22. Arridge Simon R, Betcke Marta M, Cox Ben T, Lucka Felix, Treeby Brad E. On the adjoint operator in photoacoustic tomography Inverse Problems. 2016;32:115012.

23. Treeby B. E., Cox B. T.. k-Wave: MATLAB toolbox for the simulation and reconstruction of photoacoustic wave fields Journal of Biomedical Optics. 2010;15:021314.

24. Pulkkinen Aki, Cox Ben T, Arridge Simon R, Goh Hwan, Kaipio Jari P, Tarvainen Tanja. Direct Estimation of Optical Parameters From Photoacoustic Time Series in Quantitative Photoacoustic Tomography. IEEE transactions on medical imaging. 2016;35:2497.

Author: Jenni Tick

Institute: University of Eastern Finland

Street: P.O.Box 1627

City: Kuopio

Country: Finland

Email: jenni.tick@uef.fi 